resented at the 1991 Particle Accelerator Conf., San Fran., Calif. 5/6-9/91

BNL- -45616

DE91 014025

\title{
MAIN CYCLE CONTROLS FOR THE AGS BOOSTER SYNCHROTRON*
}

\author{
B. B. Culwick, S. Yen \\ Brookhaven National Laboratory \\ Upton L.I., N.Y. 11973
}

and

\begin{abstract}
The AGS Booster is a separated function syachrotron with the main excitation coils of the dipoles and quadrupoles connected electrically in series. This circuit is driven by a complex modular power supply with curreat and voltage reference functions to obtain the desired magnetic fields as 2 function of time. The dipole cycle is defined by algebraic functions specifying the desired field profile as a function of time. These functions are processed through successive phases to convert to the signals needed to provide the power supply with one current and six voltage references. The user interface and algorithms to derive the control variables are described.
\end{abstract}

\section{INTRODUCTION}

The main magnet of the AGS Booster synchrotron is designed to operate over 2 wide range of frequencies from $7.5 \mathrm{~Hz}$ for protons to $<1 \mathrm{~Hz}$ for heavy ions. To accommodate this range while preserving precision of magnetic field value as a function of time and good power factor on the incoming power line requires a complex powier supply [1]. The design selected for the power supply makes use of six 24phese SCR controlled modules one of which is supplied with 2 current reference and all of which are supplied with voltage references. In addition to these controls, any modules not required at a given time can be by-passed by additional SCRs to eliminate their effect on power factor and ripple voltage.

The current and voltage references to the power supply modules are sequenced by vector function generators which drive digital to aralog converters [2]. The function generators are composed of two logical eatities referred to as the timing module which provides common timing to all generators, and the amplitude modules, one of which is dedicated to each function and is located at the relevant power supply. Vectors are defined by a starting value, 2 slope and a time duration. Binary controls also permii multipliers ori the slope and enable the by-passes when required.

Work performed under the nuspies of the U. S. Dept. of Energy

\section{CONTROL STRATEGY ,}

Considerations of accelerator physics, operational convenience and simplicity lod to a decision to define the main magnet cycle in terms of magnetic field versus time. To facilitate suitable parameterization, the user interface allows. graphical or numeric definition of field values and derivatives with respect to time. For reasons of tradition the units selected for the operator interface are bilogauss ad milliseconds slthough inter-program communication is in tesla and seconds. The magnetic field versus time function is then defined as a series of zones of particular algebraic form. Zone spocifications range from linear through cubic with defined derivatives or derivatives propagated from previous zones. Figure 1 shows a typical magnetic field function with a zone specification menu superimposed. From such a function it is intended that further processing be ausomatic and manual intervention is confined to verification of program actions and entry of parameters for which defaules are provided. Manual procedures are also invoked if errors or incompatibilities are encountered.

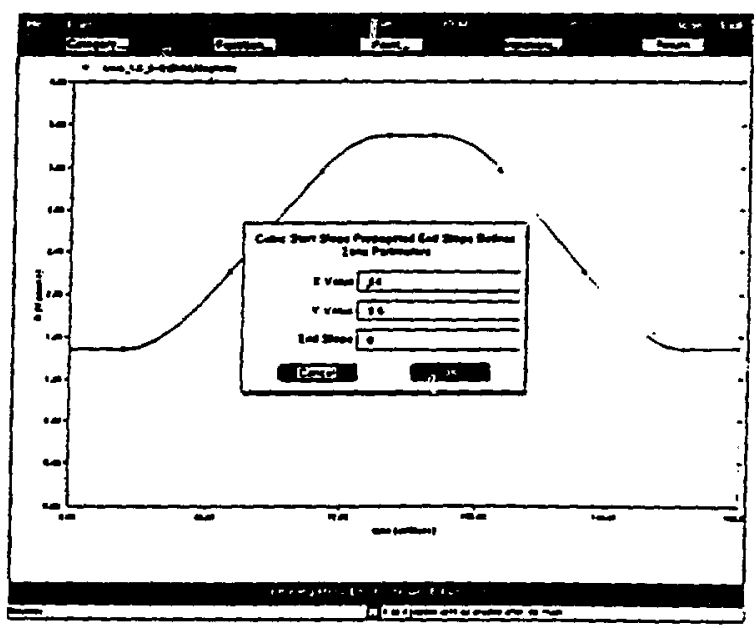

Figure 1 Magnetic Field Plot witt Zone $1:$ un 


\section{DETAILED PROCESSING}

The procedures for converting to hardware format pass through the following steps:

1) Conversion of magnetic field values to current. This procedure makes use of a calibration function expressed as a cubic spline fit to the calibration data. This facilitates subsequent differentiation of the function (see 2). No provision for hysteresis is made in the present code.

2) Calculation of the total voluge required to achieve the desired current waveform. This calculation makes use of a simple model in which the magnet is represented by a series combination of resistance and inductance. The voltage is calculated as

$$
V=R i+L \frac{d i}{d t}
$$

Af present the resistance and inductance are treated as constants but consideration has been given to making them functions of $i$ or di/dt. The time derivative of the curreat is calculated by the "chain rule"

$$
\frac{d i}{d t}=\frac{d B}{d t} \times \frac{d i}{d B}
$$

to avoid non-analytic procedures which produce discontinuities in the voltage. Finally the voltage waveform is advanced by a predetermined time to compensate for delays in the power supply. This is equivaleat to introducing a term in $\mathrm{dV} / \mathrm{dt}$ in the voltage, a procedure justified in reference [3].

3) Division of the voltage between modules is made using parameters such 25 maximum voltage per module. The order in which modules are used is defined by user selected priorities. A two step procedure avoids enabling supplies for short times or low absolute voltages.

4) A "rounding" procedure is applied to remove discontinuities in the voltage waveform at the divisions between modules. This is parametrized as the maximum slope change permitted and the time over which rounding should be applied.

This completes the algorithmic processing of the function. A typical voltage waveform is shown in Figure 2.

Any of the functions derived may be manually edited to introduce features required by testing procedures or experimental investigations. These modifications may be for onetime usage or may be preserved for future use. [4] A manually modified function is shown in figure 3 .

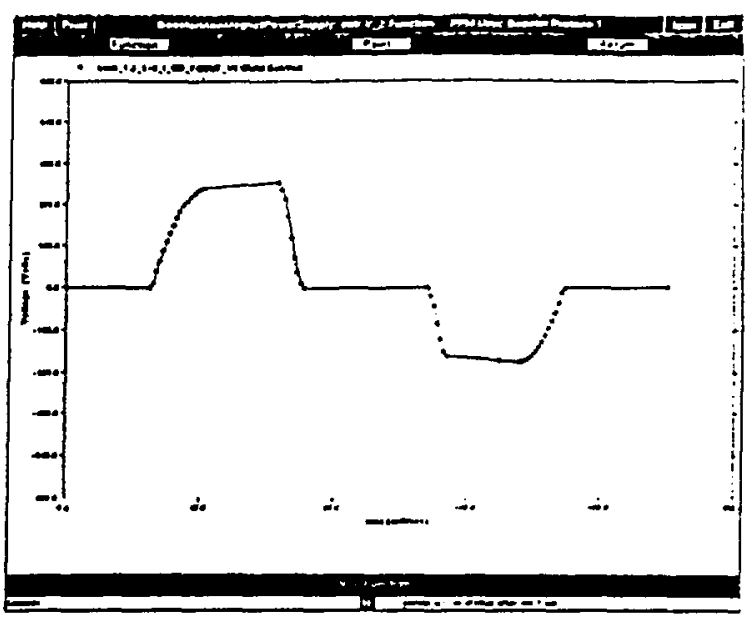

Figure 2 Typical Voltage Plot

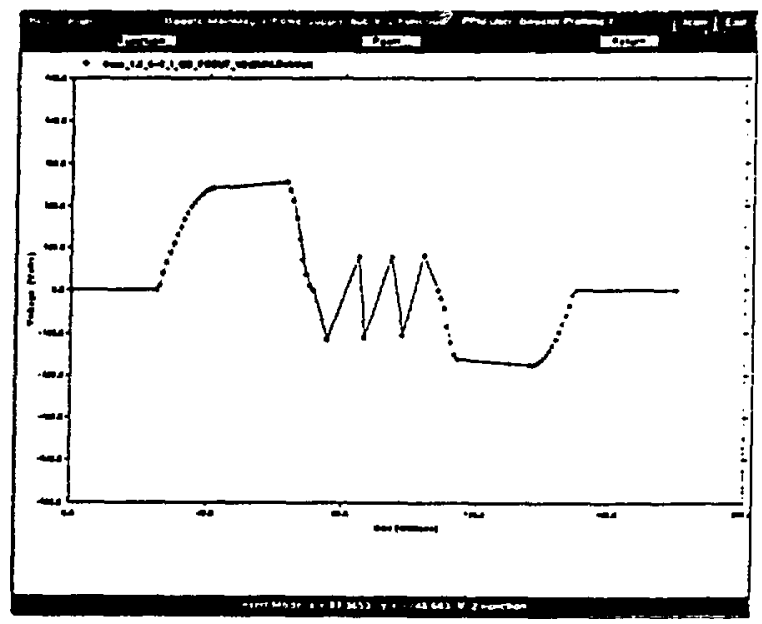

Figure 3 Manually Modified Voltage Plot

\section{EXECUTION}

Before loading a function to the Device Controller which operates the power supply in real time, the data must be converted to the vector format. The procedure consists of calculating a series of vectors of increasing length until the average value of the absolute magnirude of the deviations of the vector from the function exceeds a preset value. The previous vector is then accepted and a new vactor begun. Any vectors of zero magnitude and slope are flagged to indicate that the by-pass is to be enabled for this vector. This fitting procedure preserves the area under the curve and hence the voltage-time integral. When all functions have been fitted, the common time divisions are derived by or-ing the individual time points. This produces the common timing required by the hardware implementation. The vectors are loaded to buffers in the Device Controller. Interaction therween the 
Device Controller and the application program then allows execution of the function in a one-shot or repetitive cycle.

\section{POWER SUPPLY MONITORING}

In addition to conventional status readbacks, the power supply is equipped with 28 transient digitizers which may be triggered by faults or a timer to preserve data on power supply parameters. The control program provides for retrieval of the data from these recorders as well as from the buffers or function generator tables. These data may be presented numerically or graphically to the operator.

\section{PERFORMANCE}

The program hes been used quite exteasively in tests of the Booster Main Power Supply, the most recent with it connected to about $60 \%$ of the ring magnets. Results have been satisfactory The user interface is quite convenient and transient recorder data has been of value in recording power supply parameters. A significant modification to the magnet cycle can be accomplished in about 30 seconds.

When the Booster ring is complete, the program parameters will be optimized to model the full complement of magnets and to prepare for injoction, acceleration and extraction studies.

\section{REFERENCES}

1. A. V. Soukas et al., "AGS Booster Main Ring Power Supply System," Particle Accelerators, Vol. 29, pp. 121126, 1990.

2. N. F. Schumberg, "Dipole Power Supply Interface Specification," Private communication.

3. S. Y. Zhang, Private Communication.

4. S. Mandell, Private Communication.

\section{DISCLAIMER}

This report was prepared as an account of work sponsored by an agency of the United States Government. Neither the United States Government nor any agency thereof, nor any of their employees, makes any warranty, express or implied, or assumes any legal liability or responsibility for the accuracy, completeness, or usefulness of any information, apparatus, product, or process disclosed, or represents that its use would not infringe privately owned rights. Reference herein to any specific commercial product, process, or service by trade name, trademark, manufacturer, or otherwise does not necessarily constitute or imply its endorsement, recommendation, or favoring by the United States Government or any agency thereof. The views and opinions of authors expressed herein do not necessarily state or reflect those of the United States Government or any agency thereof. 